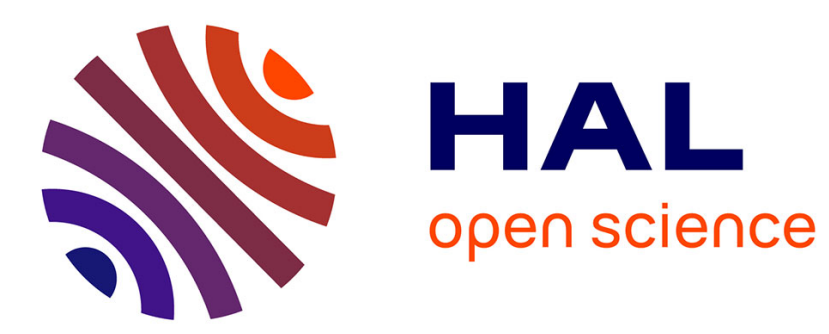

\title{
On investigation of particle dispersion by a POD approach
}

Cyrille Allery, Claudine Beghein, Aziz Hamdouni

\section{To cite this version:}

Cyrille Allery, Claudine Beghein, Aziz Hamdouni. On investigation of particle dispersion by a POD approach. International Applied Mechanics, 2008, 44 (1), pp.110-119. 10.1007/s10778-008-0025-2 . hal-00404055

\section{HAL Id: hal-00404055 \\ https://hal.science/hal-00404055}

Submitted on 31 Aug 2021

HAL is a multi-disciplinary open access archive for the deposit and dissemination of scientific research documents, whether they are published or not. The documents may come from teaching and research institutions in France or abroad, or from public or private research centers.
L'archive ouverte pluridisciplinaire HAL, est destinée au dépôt et à la diffusion de documents scientifiques de niveau recherche, publiés ou non, émanant des établissements d'enseignement et de recherche français ou étrangers, des laboratoires publics ou privés. 


\title{
ON INVESTIGATION OF PARTICLE DISPERSION BY A POD APPROACH
}

\author{
C. Allery, C. Beghein, and A. Hamdouni
}

The aim of this communication is to show the ability of POD to compute the instantaneous flow velocity when applying the Lagrangian technique to predict particle dispersion. The instantaneous flow velocity at the particle's location is obtained by solving a low-order dynamical model, deduced by a Galerkin projection of the Navier-Stokes equations onto each POD eigenfunction and it is coupled with the particle's equation of motion. This technique is applied to particle dispersion in a three-dimensional lid driven cavity. It yields a substantial decrease in computing time in comparison with LES computation and it enables treating different cases of particle dispersion

Keywords: reduced order models, proper orthogonal decomposition, particle dispersion, computational fluid dynamics

1. Introduction. Nowadays people spend a plenty of time in an indoor environment and it is thus important to predict indoor air quality in order to assess health risks. Particle dispersion is a crucial issue of indoor air quality. Indeed particle dispersion problems can be encountered when considering paint sprays, tobacco smoke, and dust inhalation. This problem can be solved numerically by using a Lagrangian technique, which requires the knowledge of the instantaneous fluid velocity at the particle's location. The instantaneous velocity can be obtained in three different ways. The first one is Direct Numerical Simulation $[3,7,17]$. The mesh should be fine enough to solve the smallest scales of motion. This approach is therefore very time consuming and cannot be applied to building simulation. Another approach is to use Reynolds Averaged Navier Stokes models to compute the time averaged turbulent flow, and to introduce a stochastic model that provides the fluctuating velocity field [8,9, 16]. These models are generally formulated for homogeneous turbulence and their extension to more complex flows is a hard task. The last approach consists in using Large Eddy Simulation (LES) to generate the instantaneous flow information [4, 19, 20]. Although the large-scale structures only are computed, applying LES to compute particle dispersion in a room is nevertheless a computationally intensive technique. Since the instantaneous velocity field and the particle dispersion are computed at the same time, the time step is imposed by the particles's equation of motion, and thus a great number of time steps (and therefore a long computing time) are necessary.

To reduce the computing time, there is another alternative still not much used. It consists in building a low-order dynamical system that provides the fluid flow dynamics. This reduced-order model can be obtained by Proper Orthogonal Decomposition (POD) that is based on the construction of an optimal "physical" basis (in energetic norm sense). A Galerkin projection of the Navier-Stokes equations onto each POD basis function yields a system of differential equations (reduced-order model) that is coupled with particles' equation of motion. This principle was already applied to investigate the motion of bubbles and particles in a turbulent boundary layer [12]. Experimental results were used to construct the POD basis. Only one POD mode was used in the wall-normal direction, and Fourier modes were used in the homogeneous spanwise and streamwise directions.

This article considers particle dispersion in a three-dimensional heterogeneous flow (lid driven cavity). The POD basis is derived by first computing the turbulent flow with Large Eddy Simulation. By comparison with LES, the ability of the POD basis and of the dynamical system to reproduce the turbulent flow is tested. The computation of particle dispersion with the 
coupled system of differential equations (low-order model and particle's equation of motion) enables a drastic reduction of computing time in comparison with LES.

2. POD Method. The Proper Orthogonal Decomposition (POD) is inspired by the Principal Component Analysis (PCA) in data processing. The main idea of PCA is to reduce the dimensionality of a data set that consists of a large number of interrelated variables, while retaining as much as possible the variation present in the data set. This is achieved by transforming the original variables into a new set of variables, the principal components, which are uncorrelated and are ordered so that the first few ones retain most of the variation present in all of the original variables. POD is thus a powerful technique for extracting basis functions that represent ensemble-averaged structures. This technique, which was introduced in fluid mechanics by Lumley [13] in 1967, has been found to be useful and efficient in approximating the dynamics of flows characterized by large-scale spatially coherent structures. As a very small number of POD modes capture most of the energy, this technique is also used to construct low-dimensional dynamic models. A Galerkin projection of the Navier-Stokes equations onto the most energetic POD modes gives a low finite-dimensional system of nonlinear ordinary differential equations (ODE) for which solution can be found with a very limited computational effort when compared to DNS or LES of a turbulent flow. Since 1988 and the pioneering paper of Aubry et al. [5] in which the motion of structures in the wall region of a flat plate turbulent boundary layer was represented by a realistic dynamical system, the construction of low-dimensional models has been considered for numerous configurations such as flows in boundary layers [14], in plane mixing layers [18], across sudden expansions [2, 10].

2.1. Proper Orthogonal Decomposition. The basic idea of POD consists in finding a "physical" basis that is optimal in an energetic sense. Thus, we search a deterministic function $\varphi$ that gives the "best" representation of the set of flow fields $u$ (assumed random and at real values) in the following sense:

$$
\overline{(u, \varphi)^{2}}=\max _{\psi \in L^{2}(\Omega)} \overline{(u, \psi)^{2}} \text { with }(\varphi, \varphi)=1,
$$

where $(\cdot, \bullet)$ denotes the inner product of $L^{2}(\Omega)$ and $\bullet$ denotes a statistic average operator; $L^{2}(\Omega)$ is the space of functions of finite energy in the flow volume $\Omega$.

From variational calculus, it follows that the above expression is equivalent to the Fredholm integral

$$
\text { Find } \lambda \in R \text { and } \varphi \text { in } L^{2}(\Omega) \text { with } \int_{\Omega} \mathrm{R}\left(x, x^{\prime}\right) \varphi\left(x^{\prime}\right) d x^{\prime}=\lambda \phi(x)
$$

representing an eigenvalue problem for $\varphi ; R$ is the spatial correlation tensor defined, with ergodicity hypothesis, by

$$
R\left(x, x^{\prime}\right)=\overline{u(x, t) \otimes u\left(x^{\prime}, t\right)} .
$$

The eigenfunctions $\varphi_{n}$ are orthogonal and all realizations of the flow $u$ are written

$$
u(x, t)=\sum_{n=1}^{+\infty} a_{n}(t) \varphi_{n}(x) \text { in } L^{2}(\Omega) \text { sense, with } a_{n}(t)=\left(u(x, t), \varphi_{n}\right) .
$$

In practice, if the sampling of the flow field is obtained by numerical simulation, the evaluation of the tensor $R$ is a very large computational task. In order to reduce the calculation, we used the Snapshots method proposed in 1987 by Sirovich [15]. In this technique, based on the fact that the eigenfunctions can be expressed in terms of the original set of data,

$$
\varphi_{n}(x)=\sum_{k=1}^{M} u\left(x, t_{k}\right) A_{n k}
$$

we must solve the matrix eigenvalue problem:

$$
\sum_{k=1}^{M} C_{k j} A_{n k}=\lambda A_{n j} \text { for } j=1, \ldots, M
$$


$A_{n k}$ denotes the constants associated with the $n$th mode, $M$ is the number of snapshots, and $C$ is the temporal correlation tensor defined by:

$$
C_{k j}=\frac{1}{M} \int_{\Omega} u\left(x, t_{j}\right) u\left(x, t_{k}\right) d x
$$

As $C$ is symmetric and positive semi-definite, all eigenvalues are real and non-negative and can be ordered as $\lambda_{1} \geq \lambda_{2} \geq \ldots \geq \lambda_{M}$. Each eigenvalue $\lambda_{n}$, taken individually, represents the energy contribution of the corresponding eigenfunction. The eigenvectors $\varphi_{n}$ are by construction incompressible (e.g., their divergence is null), satisfy the boundary conditions and can be normalized to form an orthonormal set. The main property of the POD is its ability to give the best approximation of the flow in an energetic sense [6]. The energy contained in the first NPOD modes is indeed always greater than the energy contained in any other basis such as the Fourier basis.

2.2. Low-Order Dynamical System. Usually, the first $N$ ( $N$ is small) POD modes contain most of the energy. So we can expect that a low-order dynamical system, obtained by Galerkin projection of the Navier-Stokes equations onto the most energetic eigenmodes $\varphi_{n}$, gives the flow dynamics accurately. The first step consists in approximating the flow field $u$ by keeping the first $N$ modes and ignoring the remaining modes. This approximation is written as

$$
u(x, t)=\sum_{n=1}^{N} a_{n}(t) \varphi_{n}(x)
$$

The second step consists in performing a Galerkin projection. As the continuity equation for incompressible flow is satisfied by each eigenfunction, we consider only the dimensionless momentum equation of the flow

$$
\frac{\partial u}{\partial t}+u \nabla u=-\nabla P+\frac{1}{\operatorname{Re}} \Delta u
$$

where Re and $P$ are the Reynolds number and the pressure, respectively. Introducing (8) into Eq. (9), projected onto the spatial structures $\varphi_{n}$, and taking $\varphi$ orthonormal yields

$$
\frac{d a_{n}}{d t}=\sum_{m=1}^{N} \sum_{k=1}^{N} C_{n m k} a_{m} a_{k}+\sum_{k=1}^{N} B_{n m} a_{m}+D_{n} \text { with } n=1, \ldots, N,
$$

with $C_{n m k}=-\left(\varphi_{n}, \nabla \varphi_{m} \varphi_{k}\right), B_{n m}=\left(\varphi_{n}, \Delta \varphi_{m}\right), D_{n}=-\int_{\Gamma} P \varphi_{n} n d S$, where $n$ is the outward normal on the domain $\Omega$ considered of boundary $\Gamma$.

The previous system of equations includes a term $D_{n}$ that is linked to the pressure $P$. But there is no simple expression of this term according to the expansion coefficients $\varphi_{n}$. This term should be known for each time step when integrating (10). This is quite difficult to achieve, but for a few configurations, $D_{n}$ is strictly equal to zero (in a driven cavity for instance, this case will be studied in Sec. 4, because the $\varphi_{n}$ functions are null on the boundaries) or is assumed to be zero. To overcome this difficulty if this term cannot be neglected, various approaches were considered $[1,5,14]$.

The dynamical system (10) does not include any interaction with the unresolved modes; therefore, it will not be able to dissipate enough energy and an overflow may occur. The most popular technique used to reduce this phenomenon is to assume that the influence of small scales on large scales can be modeled by an additional viscosity.

The POD method can be applied to the instantaneous field $(u$ and $P$ ) or to the fluctuating field. In this section, in order to simplify the notation, the technique has been presented for the instantaneous field. If the fluctuating field is considered, the dynamical system is slightly modified. It is obtained by decomposing the instantaneous velocity and pressure fields into a mean component $\bar{\bullet}$, with the fluctuating part of the velocity $\bullet^{\prime}$ expressed according to the POD basis. This yields

$$
\begin{gathered}
u(x, t)=\bar{u}(x)+u^{\prime}(x, t) \approx \bar{u}(x)+\sum_{n=1}^{N} a_{n}(t) \varphi_{n}(x), \\
P(x, t)=\bar{P}(x)+P^{\prime}(x, t) .
\end{gathered}
$$




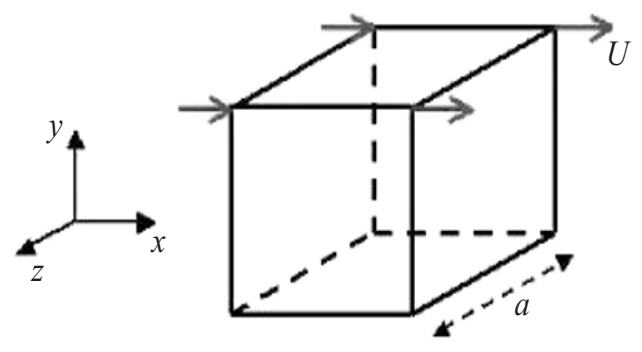

Fig. 1. Geometry of the lid driven cavity (the coordinate system is located in the center of the cavity)

These expressions are then introduced into the Navier-Stokes equations. The expression for the coefficients is detailed in [1,14].

3. Particle Model. A monodisperse aerosol in an isothermal flow is considered here. The particles are solid and spherical. Aerosols in buildings are dilute, the particles do not modify the flow and do not coagulate, a one-way coupling approach is thus used. The Lagrangian approach is employed in order to compute the trajectories of particles. A large number of particles should be released in the flow in order to obtain statistically independent results. Since the density ratio (particle density versus gas density) is rather high (about 2000), the particles are mainly submitted to the gravity and drag forces. Other forces such as the buoyancy force, the added mass effect, and the Basset force are negligible. In addition, the particles do not exhibit a random motion due to Brownian effects, because the particle diameters in this study are greater than one micron. The particle equation of motion is therefore

$$
\rho_{p} \frac{\pi d_{p}^{3}}{6} \frac{d u_{p}}{d t}=\frac{\pi d_{p}^{3}}{6} \rho_{p} g+\frac{\pi d_{p}^{2}}{8} \rho C_{d}\left\|u-u_{p}\right\|\left(u-u_{p}\right)
$$

where $\rho_{p}, d_{p}, u_{p}$ are the density, diameter and velocity of the particle; $g$ is the gravitational acceleration; $\rho$ is the fluid density; and $u$ is the instantaneous fluid velocity at the particle's location. According to Hinds [11], the drag coefficient can be calculated as a function of the particle Reynolds number $\mathrm{Re}_{p}$ in the following manner:

$$
C_{d}= \begin{cases}\frac{24}{\operatorname{Re}_{p}}, & \text { if } \operatorname{Re}_{p}<1, \\ 0.44 & \text { if } \operatorname{Re}_{p}>1000 \\ \frac{24}{\operatorname{Re}_{p}}\left(1+\frac{\operatorname{Re}_{p}^{2 / 3}}{6}\right), & \text { if } 1<\operatorname{Re}_{p}<1000\end{cases}
$$

with $\operatorname{Re}_{p}=\frac{\left\|u-u_{p}\right\|}{v}$ and $v$ is the kinematic fluid viscosity. Equation (12) is solved at each time step, for each particle. Integrating this equation over time yields the particle velocity. The particle position at each time step is obtained by integrating

$$
u_{p}=\frac{d x_{p}}{d t}
$$

To sum up, in this work, the instantaneous fluid velocity at the particle's location is provided by the Proper Orthogonal Decomposition method, so combining Eqs. (10), (12), and (14) yields the following coupled problem:

$$
\frac{d \boldsymbol{a}}{d t}=F(\boldsymbol{a}, t), \quad \frac{d u_{p}}{d t}=G\left(\boldsymbol{a}, u_{p}, t\right), \quad \frac{d x_{p}}{d t}=u_{p},
$$

where $\boldsymbol{a}=\left(a_{1}, \ldots, a_{n}\right)$. This set of differential equations is solved by the Runge-Kutta method.

4. Results. The application case considered is particle dispersion in an isothermal flow in a cubic lid driven cavity filled with air, whose side $a$ is equal to $1 \mathrm{~m}$. (Fig. 1). The fluid motion in the cavity is enhanced by the motion of the upper horizontal wall. The wall velocity $U$ is $0.15 \mathrm{~m} / \mathrm{sec}$, which corresponds to a Reynolds number based on the cavity height of 10,000 . The turbulent flow was computed with the Smagorinsky LES model. The flow domain is discretized into a nonuniform mesh of 


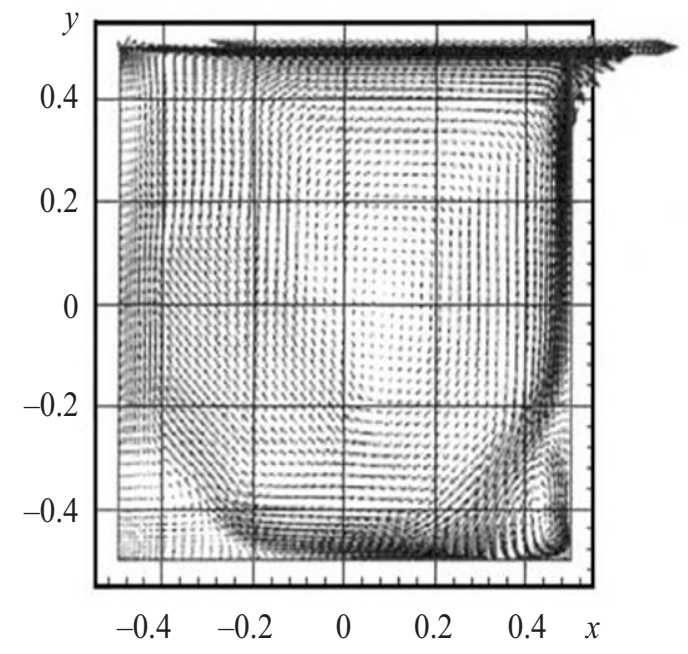

$a$

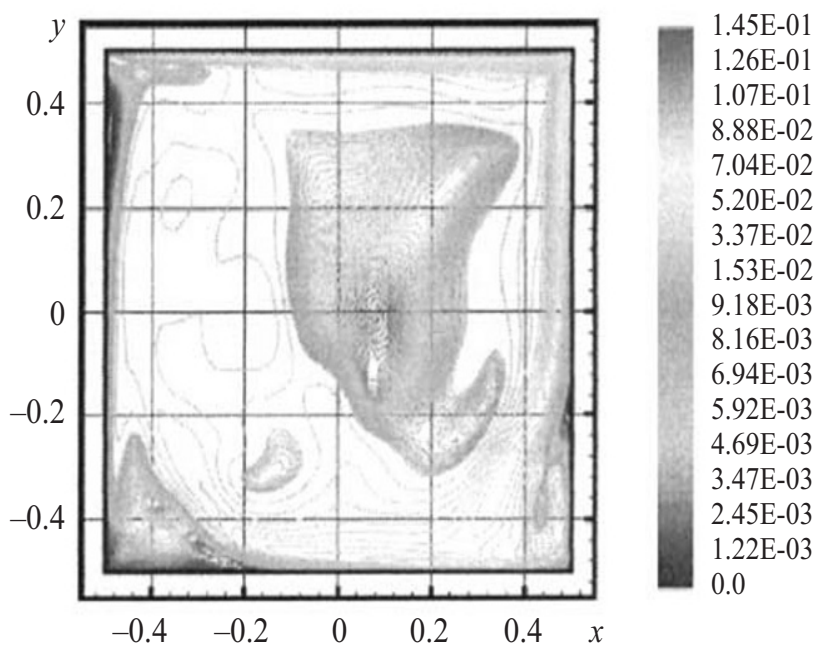

$b$

Fig. 2. Mean velocity field in the vertical mid plane $z=0$, obtained by LES: vectors $(a)$, isocontours of velocity magnitude $(\mathrm{m} / \mathrm{sec})(b)$

TABLE 1. Eigenvalues, cumulative energy, residuals

\begin{tabular}{|c|c|c|c|}
\hline Mode $n$ & $\lambda_{n}$ & $100 \times E_{c}$ & $\operatorname{res}_{N}$ \\
\hline 1 & $4.613 \mathrm{E}-06$ & 85.24 & $2.313 \mathrm{E}-01$ \\
\hline 2 & $6.755 \mathrm{E}-07$ & 97.73 & $5.331 \mathrm{E}-02$ \\
\hline 3 & $9.939 \mathrm{E}-08$ & 99.56 & $1.439 \mathrm{E}-02$ \\
\hline 4 & $1.899 \mathrm{E}-08$ & 99.81 & $3.947 \mathrm{E}-03$ \\
\hline
\end{tabular}

$58 \times 58 \times 58$ cells. A cloud of particles (density $\rho_{P}=2,000 \mathrm{~kg} / \mathrm{m}^{3}$ ) is released in the upper part of the cavity close to the upper horizontal wall $\left(0.03 \mathrm{~m}<x_{p}<0.07 \mathrm{~m}, 0.48 \mathrm{~m}<y_{p}<0.49 \mathrm{~m},-0.05 \mathrm{~m}<z_{p}<0.05 \mathrm{~m}\right)$. The initial velocity of particles is equal to the instantaneous fluid velocity. The time step chosen for particle computation is $0.0002 \mathrm{sec}$. Particles are attached to the wall when they contact the surfaces. Since particles are injected in the vicinity of the vertical mid plane $z=0$ of the cavity, we present the mean velocity field in this plane in Fig. 2. It consists of a big noncentered clock-wise recirculation region, two counter-clock-wise small recirculation regions in the lower right and left corners, and a smaller one in the upper left corner. In addition, the strong velocity regions are located close to the walls.

4.1. POD Application. Once the LES computation of the turbulent flow was converged, 100 snapshots of fluctuating velocity $(M=100)$ fields were recorded every $0.1 \mathrm{sec}$ to build the POD basis.

In Table 1, the eigenvalues $\lambda_{n}$ relative to mode $n$, the cumulative energy $E_{c}$ contained in the first $N$ POD modes, and a normalized residual showing the ability of the first $N$ POD modes to reproduce the flow velocity are presented. These quantities are defined according to

$$
E_{c}=\sum_{i=1}^{N} \lambda_{i} / \sum_{i=1}^{M} \lambda_{i} \quad \operatorname{res}_{N}=\|\beta\|_{\infty} /\|\eta\|_{\infty}
$$

with $\beta^{2}=\int_{\Omega}\left[u(x, t)-\sum_{n=1}^{N} a_{n}(t) \varphi_{n}(x)\right]^{2} d x$ and $\eta^{2}=\int_{\Omega}[u(x, t)]^{2} d x$. Here $\|\cdot\|_{\infty}$ is the infinite norm. 

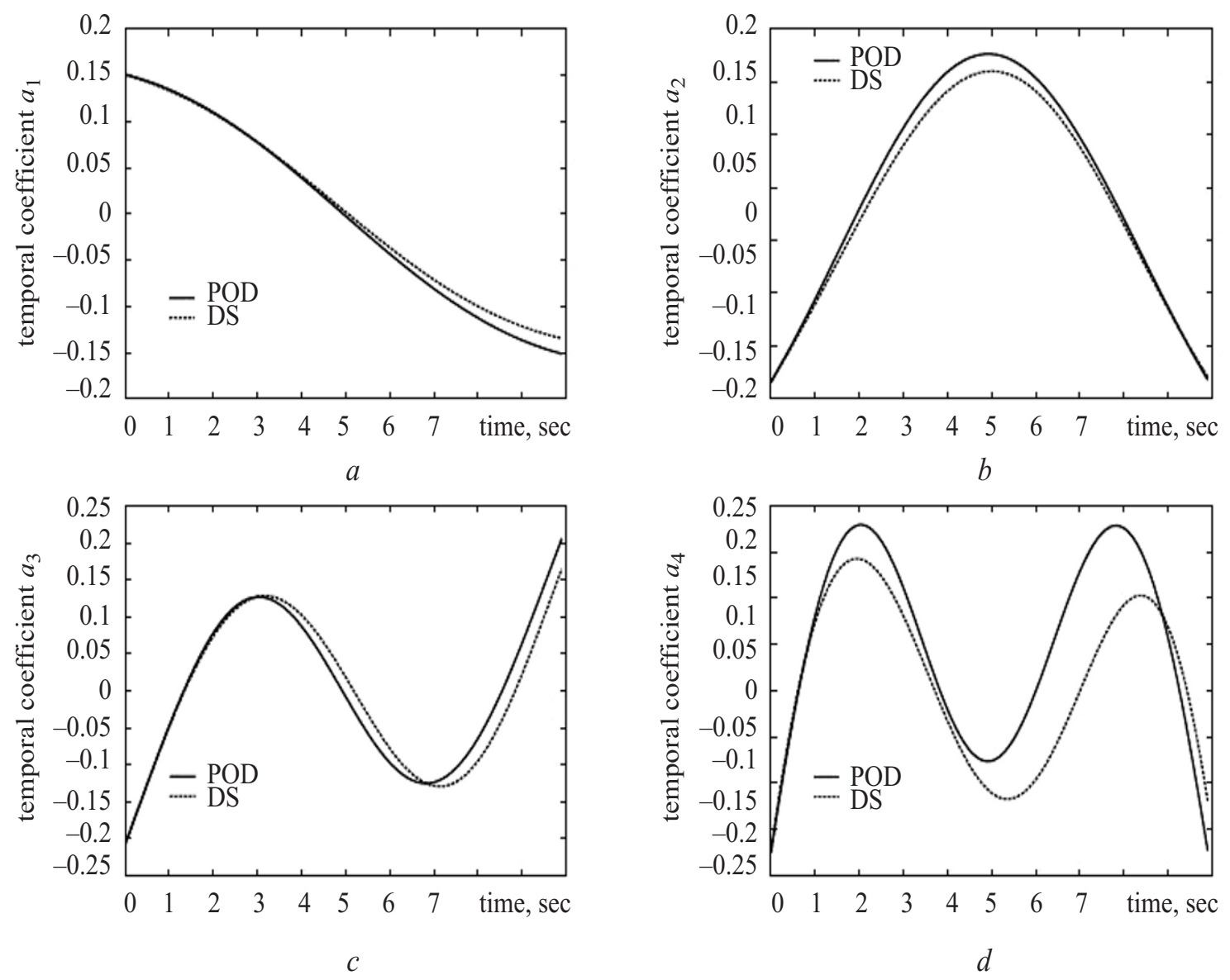

Fig. 3. Temporal evolution of the four modes $a_{1}(t), \ldots, a_{4}(t)$ : mode $1(a)$, mode $2(b)$, mode $3(c)$, mode $4(d)$

In Table 1, we can notice that one mode contains $85 \%$ of energy and with four modes the fluctuating energy of the signal is almost captured. It also indicates that only four modes are necessary to obtain a residual less than $4 \times 10^{-3}$ (which corresponds to a relative error of $1.6 \times 10^{-5}$ ). With four modes, the fluctuating velocities are hence well reconstructed. Therefore, the low-order system, used to compute the flow dynamics and particle dispersion, is obtained with four modes.

4.2. Dynamical System (DS). Since the dynamical behavior of the flow is obtained with the $a_{n}(t)$ coefficients (Eq. (8)) it is interesting to focus on the quality of these coefficients. Figure 3 shows the temporal evolution of the four modes $a_{1}(t), \ldots, a_{4}(t)$ during the sampling time used for the snapshot method.

In this figure, POD corresponds to the reference solution obtained by projecting the fluctuating LES velocity field onto the spatial structure $\varphi_{n}$ (Eq. (4)) and DS is the solution obtained by solving the dynamical system (Eq. (10)). In order to account for the effect of the unresolved modes on the resolved ones, a viscosity of $2.7 \times 10^{-5}$ was added to the kinematic viscosity of the dynamical system. It can be noticed that the temporal evolution of the reference functions POD are properly recovered from the dynamical system.

Figure 4 gives the fluctuating velocity field obtained by LES and by the low order dynamical system at time $t=9 \mathrm{sec}$. The velocity vectors show that there is no difference between the reference solution and the reconstructed fluctuating velocity field. But if we examine the velocity magnitude in detail (Fig. 5), we can see that there are small differences between these two fields. Similar results and remarks not presented here are obtained for the other sampling times. For a quantitative study of particle dispersion, attention should be paid to the influence of the neglected modes as done, for example, by Wang and Squires [19]. For this communication focused on the ability of POD to predict particle dispersion by a low-order model, we nevertheless consider that the dynamical behavior of the flow is properly predicted by "our" low-order system.

4.3. Particle Dispersion. Although the POD basis was built by using $10 \mathrm{sec}$ of snapshots, particle dispersion was computed with the low-order dynamical system for a duration of $120 \mathrm{sec}$.

In the first series of simulations, a cloud of $5 \mu \mathrm{m}$ diameter particles was released in the upper part of the cavity. Since POD enables to compute the instantaneous flow with a reduced computational effort, it is "easy" to treat various cases of particle dispersion (various diameters and particle's injection), in comparison with LES or DNS. Indeed, the first step was to vary the 

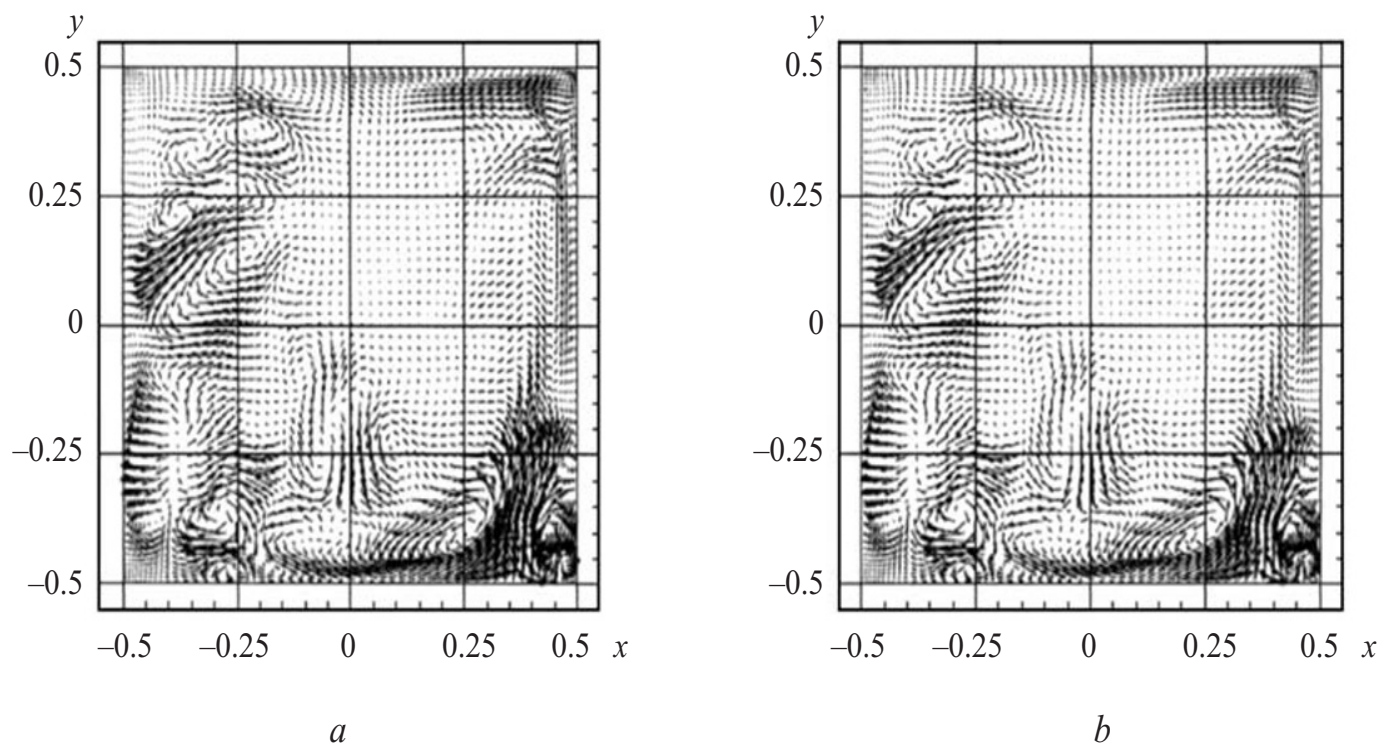

Fig. 4. Fluctuating velocity vectors at time $t=9 \sec$ in the $z=0$ plane: reference solution LES $(a)$, obtained by solving DS (4 modes) $(b)$

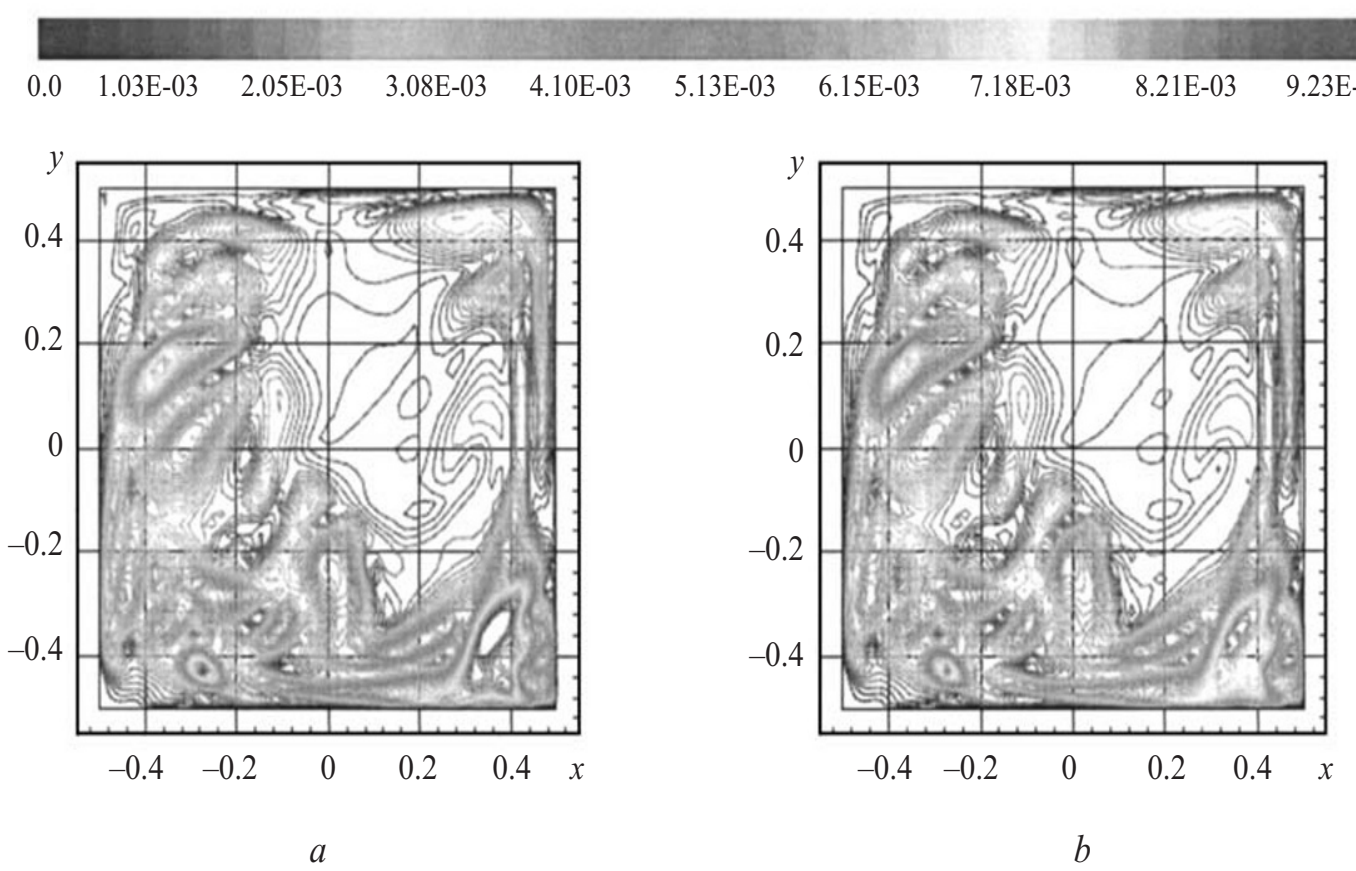

Fig. 5. Isocontours of fluctuating velocity magnitude at time $t=9 \mathrm{sec}$ in the $z=0$ plane: reference solution LES $(a)$, obtained by solving DS (4 modes) $(b)$

number of particles released in the air flow in order to obtain statistically independent results. Figure 6 depicts the percentage of 5,000 particles stuck on the lower horizontal wall according to time. It shows that injecting 5,000 particles in the flow yields statistically independent results.

Figure 7 presents the temporal evolution of the particles' cloud. One can notice that, since particles are driven by air flow with a strong velocity close to the wall, some of them are stuck on the floor due to the combined effects of inertia and gravity. Nevertheless, since these particles are light, many of them follow the jet path, go to the small recirculation regions in the lower left corner of the cavity and are lifted towards the upper part of the cavity.

In the second series of simulations, a cloud of 5,000 $10 \mu \mathrm{m}$ diameter particles was released in the same location as in the previous simulations. In Fig. 8, the temporal evolution of the particles' cloud in the cavity is given. Since these particles are more inert than in the previous case, it takes a longer time for these particles to get to the upper right corner of the cavity. Moreover, due to the gravity force, these particles cannot be lifted by the left ascending flow. They deposit in a smaller duration than the $5 \mu \mathrm{m}$ particles (about $85 \mathrm{sec}$ ). 


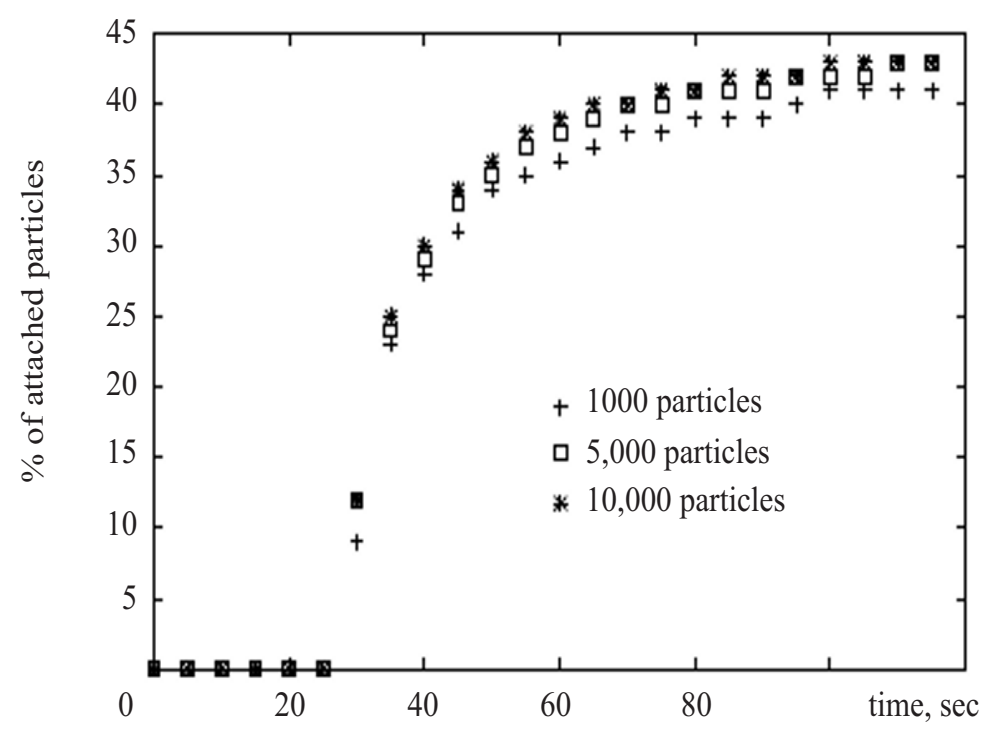

Fig. 6. Percentage of attached particles according to time $($ diameter $=5 \mu \mathrm{m})$
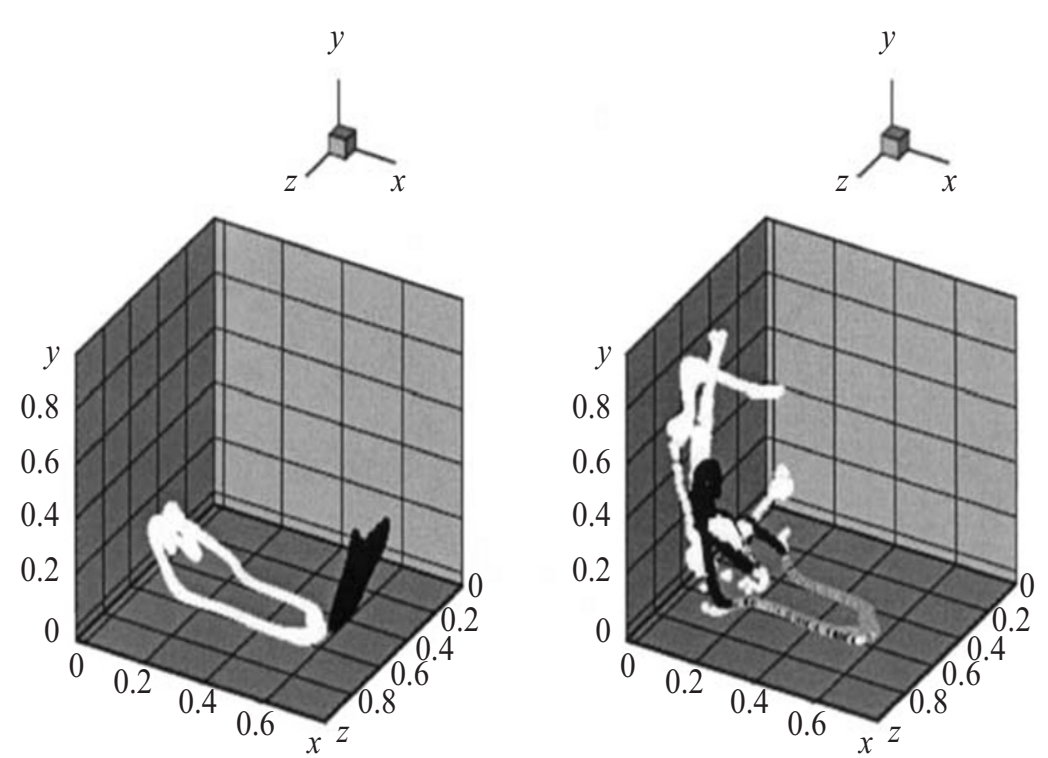

Fig. 7. Temporal evolution of the $5 \mu \mathrm{m}$ particles' cloud in the cavity: Left: $20 \mathrm{sec}$ (black), $64 \mathrm{sec}$ (white); Right: $80 \mathrm{sec}$ (black), $120 \mathrm{sec}$ (white) since particles' injection

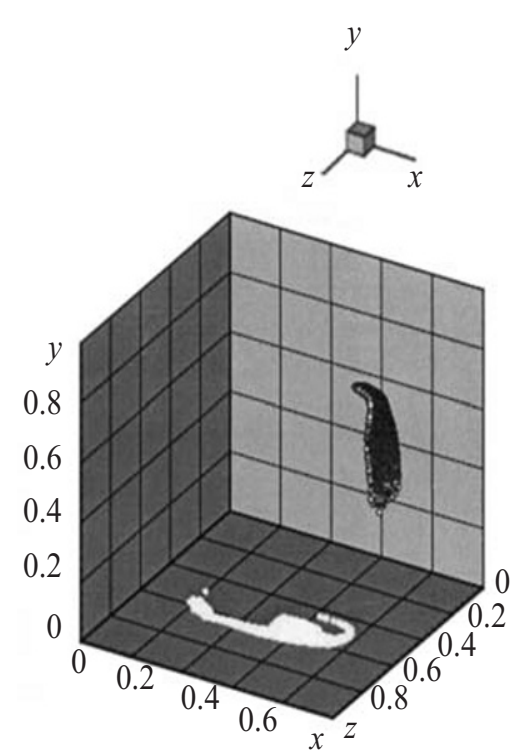

Fig. 8. Temporal evolution of the $10 \mu \mathrm{m}$ particles' cloud in the cavity: $20 \mathrm{sec}$ (black), $64 \mathrm{sec}$ (white) since particles' injection)

4.4. Algorithm and POD/Particle Advantages. To sum up, a full simulation of a POD/particle approach requires the following steps.

1. Perform an LES or DNS simulation for the sampling time $T_{\text {sample }}$, to get the POD basis.

2. Compute the $C_{n m k}, B_{n m}$, and $D_{n}$ coefficients of the dynamical system (10).

3. Solve the coupled problem POD/particle (15) for a duration $T \gg T_{\text {sample. }}$

The first and second steps are done once for a given flow.

The advantage of this approach is that if another case of particle dispersion (diameter, density, position of particles injection...) is dealt with for this flow, the third step only must be done (since the POD basis and the coefficients of the dynamical system remain the same). If the LES or DNS approach is employed, whatever the particle dispersion case simulated, the flow must be re-computed, and this requires a great amount of time. Moreover, POD enables to significantly decrease the computing time in comparison with LES. Let us consider for instance the dispersion of 1000 particles for a duration of $120 \mathrm{sec}$. The time step 
imposed by the integration of the particles' motion equation is $\Delta t_{\text {part }}=2 \times 10^{-4} \mathrm{sec}$. Step 1 of the algorithm (sample period) was done for a duration $T_{\text {sample }}=10 \mathrm{sec}$ with a time step $\Delta t_{\text {sample }}=0.01 \mathrm{sec}$. This step requires $40 \mathrm{~min}$ computations for 10 processors. Computations of step 2 lasted only $10 \mathrm{~min}$. And computations of step 3, were performed in $20 \mathrm{~h}$. The full simulation of this POD/Particle problem took thus about $24 \mathrm{~h}$ computations.

Solving the coupled problem LES/particle for a duration of $120 \mathrm{sec}$ with a time step $\Delta t_{\text {part }}=2 \times 10^{-4} \mathrm{sec}$ considerably increases the computing time. We estimate (with the computations performed for the sampling procedure) that the computing time for an LES/particle computation would be more than one week.

5. Conclusion. In this communication, we presented a numerical study of particle dispersion in a lid driven cavity. The behavior of the dynamical system during the sampling time necessary for the snapshot method was validated by comparison with the LES results. A drastic decrease of the computing time was highlighted as well as the possibility to treat different cases of particle dispersion with a minimum effort. This case has shown the ability of POD to qualitatively predict particle dispersion for one way coupling cases. The next step of our work is to include a model to account for the effect of the nonresolved modes on the particles. Another perspective is to treat a real configuration encountered in a building.

Acknowledgment. The authors wish to express their thanks to the CINES (Centre Informatique National de l'Enseignement Supérieur). The computations were performed with the IBM SP of the CINES.

\section{REFERENCES}

1. C. Allery, Contribution à l'identification des bifurcations et à l'étude des écoulements fluides par des systèmes dynamiques d'ordre faible (P.O.D.), Ph. D. Thesis, University of Poitiers, France (2002).

2. C. Allery, S. Guérin, A. Hamdouni, and A. Sakout, "Experimental and numerical POD study of the Coanda effect used to reduce self-sustained tones," Mechanics Research Communication, 31, No. 1, 105-120 (2004).

3. V. Armenio and V. Fiorotto, "The importance of the forces acting on particles in turbulent flows," Physics of Fluids, 13, No. 8, 2437-2440 (2001).

4. V. Armenio, U. Piomelli, and V. Fiorotto, "Effect of the subgrid scales on particle motion," Physics of Fluids, 11, No. 10, 3030-3042 (1999).

5. N. Aubry, P. Holmes, J. L. Lumley, and E. Stone, "The dynamics of coherent structures in the wall region of a turbulent boundary layer," J. Fluid Mech., 192, 115-173 (1988).

6. G. Berkooz, P. Holmes, and J. L. Lumley, "The proper orthogonal decomposition in the analysis of turbulent flows," Ann. Rev. Fluid Mech., 25, 539-575 (1993).

7. M. Chen and J. B. McLaughlin, "A new correlation for the aerosol deposition rate in vertical ducts," J. Colloid Interf. Sci., 169, 437-455 (1995).

8. P. Desjonquères, A. Berlemont, and G. Gouesbet, "A Lagrangian approach for the prediction of particle dispersion in turbulent flows," J. Aerosol Sci., 19, No. 1, 99-103 (1988).

9. A. D. Gosman and E. Ioannides, "Aspects of computer simulation of liquid-fuelled combustors," in: Paper 81-0323 presented at the AIAA 19, Aerospace Science Mtg., St Louis, MO.

10. H. Gunes, "Low dimensional modeling of non-isothermal twin-jet flow," Int. Communications in Heat and Mass Transfer, 29, No. 1, 77-86 (2002).

11. W. C. Hinds, Aerosol Technology: Properties, Behaviour, and Measurement of Airborne Particles, John Wiley and Sons, New York (1982).

12. I. A. Joia, T. Ushijima, and R. J. Perkins, "Numerical study of bubble and particle motion in a turbulent boundary layer using proper orthogonal decomposition," Appl. Sci. Research, 57, 263-277 (1997).

13. J. L. Lumley, "The structure of inhomegeneous turbulent flows," in: Yaglom and Tararsky (eds.), Atmospheric Turbulence and Radio Wave Propagation (1967), pp. 166-178.

14. D. Rempfer, "Investigations of boundary layer transition via Galerkin projections on empirical eigenfunctions," Physics of Fluids, 8, No. 1, 175-188 (1996).

15. L. Sirovich, "Turbulence and the dynamics of coherent structures, Part 1: Coherent structures. Part 2: Symmetries and transformations. Part 3: Dynamics and scaling," Quarterly of Applied Mathematics, 45, 561-590 (1987). 
16. M. Sommerfeld, G. Kohnen, and M. Rüger, "Some open questions and inconsistencies of Lagrangian particle dispersion models," Paper 15.1 presented at the 9th Symp. on Turbulent Shear Flows, Kyoto, August (1993).

17. D. G. Thakurta, M. Chen, J. B. McLaughlin, and K. Kontomaris, "Thermophoretic deposition of small particles in a direct numerical simmulation of turbulent channel flow," Int. J. Heat and Mass Transfer, 41, 4167-4182 (1998).

18. L. Ukeiley, L. Cordier, R. Manceau, J. Delville, M. Glauser, and J. P. Bonnet, "Examination of large-scale structures in a turbulent plane mixing layer. Part 2. Dynamical systems model," J. Fluid Mech., 441, 67-108 (2001).

19. Q. Wang and K. D. Squires, "Large eddy simulation of particle deposition in a vertical turbulent channel flow," Int. J. Multiphase Flow, 22, No. 4, 667-683 (1996).

20. F. Yeh and U. Lei, "On the motion of small particles in a homogeneous isotropic turbulent flow," Physics of Fluids, $\mathbf{3}$, No. 11, 2571-2586 (1991). 
